\title{
Erratum to: Attenuation of $A \beta$-induced neurotoxicity by thymoquinone via inhibition of mitochondrial dysfunction and oxidative stress
}

\author{
Andleeb Khan - Kumar Vaibhav • Hayate Javed • Mohd. Moshahid Khan • \\ Rizwana Tabassum • Md. Ejaz Ahmed P Pallavi Srivastava • Gulrana Khuwaja • \\ Farah Islam • Mohd. Saeed Siddiqui $\cdot$ Mohammed M. Safhi $\cdot$ Fakhrul Islam
}

Published online: 17 January 2013

(c) Springer Science+Business Media New York 2013

\section{Erratum to: Mol Cell Biochem (2012) 369:55-65 \\ DOI 10.1007/s11010-012-1368-x}

In the original article, the surname of M. M. Shafi has been misspelled. The correct spelling of his name is M. M. Safhi.

Everything else in the paper remains correct.

The online version of the original article can be found under doi: 10.1007/s11010-012-1368-x.

A. Khan - K. Vaibhav $\cdot$ H. Javed · Mohd. Moshahid Khan . R. Tabassum - Md. E. Ahmed - P. Srivastava - G. Khuwaja · Mohd. Saeed Siddiqui · F. Islam

Neurotoxicology Laboratory, Department of Medical Elementology and Toxicology (Fund for the Improvement of Science and Technology Sponsored by DST and Special Assistance Programme Sponsored by UGC), Jamia Hamdard (Hamdard University), Hamdard Nagar, New Delhi 110062, India

Present Address:

Mohd. Moshahid Khan

Department of Neurology, Carver College of Medicine,

University of Iowa, Iowa City, IA 52242, USA

Present Address:

P. Srivastava

Department of Neurology, UMDNJ, Robert Wood Johanson

Medical School, Brunswick, NJ, USA
Present Address:

G. Khuwaja · M. M. Safhi · F. Islam ( $\square)$

Neuroscience and Toxicology Unit, Faculty of Pharmacy, Jazan

University, Jazan, Kingdom of Saudi Arabia

e-mail: fislam2001@yahoo.co.in

F. Islam

Department of Biotechnology, Faculty of Pharmacy, Jamia

Hamdard (Hamdard University), Hamdard Nagar, New Delhi 110062, India 